ACCEPTED MANUSCRIPT

\title{
Steering surface plasmons with a graded index dielectric medium
}

To cite this article before publication: Victor Pacheco-Peña et al 2018 J. Phys. D: Appl. Phys. in press https://doi.org/10.1088/1361-6463/aae3a5

\section{Manuscript version: Accepted Manuscript}

Accepted Manuscript is "the version of the article accepted for publication including all changes made as a result of the peer review process, and which may also include the addition to the article by IOP Publishing of a header, an article ID, a cover sheet and/or an 'Accepted Manuscript' watermark, but excluding any other editing, typesetting or other changes made by IOP Publishing and/or its licensors"

This Accepted Manuscript is @ 2018 IOP Publishing Ltd.

During the embargo period (the 12 month period from the publication of the Version of Record of this article), the Accepted Manuscript is fully protected by copyright and cannot be reused or reposted elsewhere.

As the Version of Record of this article is going to be / has been published on a subscription basis, this Accepted Manuscript is available for reuse under a CC BY-NC-ND 3.0 licence after the 12 month embargo period.

After the embargo period, everyone is permitted to use copy and redistribute this article for non-commercial purposes only, provided that they adhere to all the terms of the licence https://creativecommons.org/licences/by-nc-nd/3.0

Although reasonable endeavours have been taken to obtain all necessary permissions from third parties to include their copyrighted content within this article, their full citation and copyright line may not be present in this Accepted Manuscript version. Before using any content from this article, please refer to the Version of Record on IOPscience once published for full citation and copyright details, as permissions will likely be required. All third party content is fully copyright protected, unless specifically stated otherwise in the figure caption in the Version of Record.

View the article online for updates and enhancements. 


\title{
dielectric medium
}

Steering surface plasmons with a graded index

\author{
Victor Pacheco-Peña ${ }^{1^{*}}$ and Miguel Beruete ${ }^{2,3^{*}}$ \\ ${ }^{1}$ Emerging Technologies and Materials group, School of Engineering, Newcastle University, Newcastle \\ Upon Tyne, NE1 7RU, UK \\ ${ }^{2}$ Antennas Group-TERALAB, Universidad Publica de Navarra, Campus Arrosadia, 31006 Pamplona, \\ Spain \\ ${ }^{3}$ Institute of Smart Cities, Public University of Navarra, 31006, Pamplona, Spain
}

E-mail: victor.pacheco-pena@newcastle.ac.uk and miguel.beruete@unavarra.es

Received $\operatorname{xxxxxx}$

Accepted for publication $\mathrm{xxxxxx}$

Published xxxxxx

\begin{abstract}
The arbitrary control of surface plasmons (SP) propagation has become an intense research topic for several decades. This is due to the fact that they can be used in a variety of fields such as optical trapping, nanoantennas and medical applications. In this communication, the graded index technique is applied in the design of several steerers able to tailor the direction of propagation of the incident SPs by simply changing the height of a dielectric block on top of a semi-infinite metal slab. The design procedure is shown and the structures are numerically evaluated demonstrating a good agreement with the analytical calculations, with the SPs deflected at the design angles ( $\theta$ ranging from $10^{\circ}$ to $60^{\circ}$ ) with a wide bandwidth steering of $60 \mathrm{~nm}$ around the design value $(633 \mathrm{~nm})$.
\end{abstract}

Keywords: Surface plasmons, steering surface plasmons, plasmonics, effective medium theory

\section{Introduction}

Nanophotonics deals with the study of the behavior of light at the nanometer scale[1-4]. In this context, the pursue of an efficient control of the interaction of light with metal particles or films has given rise to the emerging field of plasmonics[5,6], mainly concerned with the interaction process between the electromagnetic (EM) radiation and conduction electrons in metals when these can be modelled as dispersive media with a permittivity following the Drude or Drude-Lorentz model.

Plasmonics borrows the name from surface plasmons (SPs) which are surface waves that propagate along the interface between a dielectric and a conductor and which are evanescently confined in the perpendicular direction [7]. The existence of SPs is directly related to the dispersive permittivity of metals at optical frequencies, since they can be excited when the real part of the permittivity of both media have different signs (positive for the dielectric and negative for the metal) but with similar magnitude.

Nowadays, the study of SPs has become an intense field of research and many efforts have been dedicated to get control over their propagation. Different applications have been proposed using SPs such as focusing [8-12], plasmon induced transparency [13] waveguides and filters [9,14,15], nanoantennas [16-20], and optical trapping[16], to name a few. Within this realm, a prominent research field is to tailor the propagation direction of SPs [21-27].

In this work, the graded index (GRIN) technique is applied in the design of GRIN-SPs steerers using a dielectric block on top of a semi-infinite metal slab. First, the analytical formulation for the effective refractive index of the air-metal and air-dielectric-metal regions is shown. Then, several GRIN-SP steerers are designed to deflect the incoming SPs to 
an arbitrary output angle by changing the height (or equivalently the effective refractive index) of the dielectric block. Moreover, as an alternative to changing the height, it is shown that SP steerers can be designed by tuning the length of the dielectric block to achieve the required phase profile at the output of the nanostructure.

\section{Design}

A schematic representation of the proposed nanostructure is shown in figure $1(\mathrm{a}, \mathrm{b})$. It consists of a dielectric block on top of a semi-infinite metal slab made of gold $(\mathrm{Au})$ with its dispersive permittivity modeled following Johnson and Christy experimental data[28]. The GRIN dielectric nanostructure, immersed in air $\left(n_{l}=1\right)$, is designed at the optical communications wavelength of $\lambda_{0}=633 \mathrm{~nm}$, with a height and length represented by $l_{y}$ and $l_{z}$, respectively. Silicon Nitride $\left(\mathrm{Si}_{3} \mathrm{~N}_{4}\right)$ is used as the material for the dielectric block with a refractive index of $n_{2} \sim 1.99$ at the design wavelength. All the numerical simulations here presented are done using the transient solver of the commercial software CST Microwave Studio $^{\circledR}$. The SPs are excited at the back of the GRIN medium by using a narrow waveguide[29,30]. With this setup, the SPs propagate along the interface between the waveguide and the air-Au region in the positive $z$ direction with magnetic field parallel to the $x$-axis and electric field lying along the $y$-and $z$-axes.
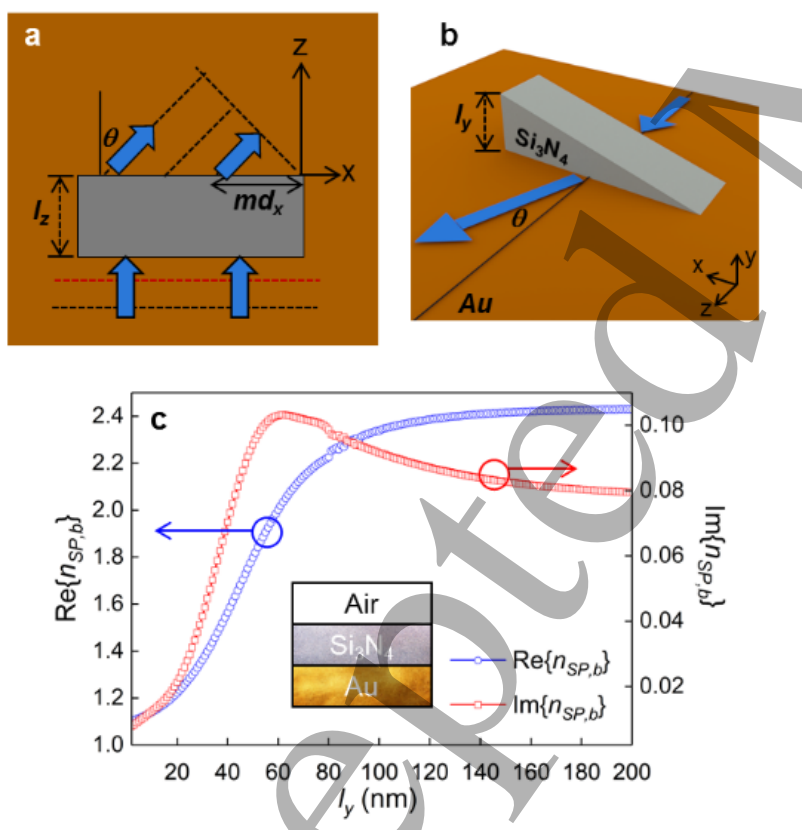

Figure. 1. Schematic representation of the GRIN-SP dielectric steerer: (a) top and (b) perspective view. The dimensions of the $\mathrm{Si}_{3} \mathrm{~N}_{4}$ wedge are $l_{y}$ and $l_{z}$ along the $y$ and $z$ axes, respectively with a total width along the $x$ axis of $3.55 \lambda_{0}$ at the wavelength of $\lambda_{0}=633 \mathrm{~nm}$. (c) Real (blue) and imaginary (red) parts of the effective refractive index of the region air$\mathrm{Si}_{3} \mathrm{~N}_{4}-\mathrm{Au}$ as a function of $l_{y}$ at the design wavelength of $633 \mathrm{~nm}$.
Let us first study the propagation properties of SPs under the proposed configuration shown in figure $1(a, b)$. The process to analyse such structure has been used in the design of plasmonic applications such as focusing elements[25$27,29,30]$ and the basic concepts are presented here for completeness. As observed in the figure, there are two different regions based on the different materials involved: a) air-Au and b) air- $\mathrm{Si}_{3} \mathrm{~N}_{4}-\mathrm{Au}$. The features of the SPs within these two regions can be described in terms of the effective propagation constant $(\beta)$ which can be then translated into an effective refractive index $\left(n_{S P}\right)$.

For the case of the air-Au region, the effective propagation constant can be calculated by using the well-known expression $\beta_{a}=k_{0}\left[\left(n_{1}^{2} n_{A u^{2}}{ }^{2}\right) /\left(n_{1}^{2}+n_{A u^{2}}{ }^{2}\right)\right]^{1 / 2}$ where $k_{0}=2 \pi / \lambda_{0}$ is the wavenumber in free-space, $n_{\perp}$ is the refractive index of the dielectric in the first region (air in this case) and $n_{A u}$ is the refractive index of the semi-infinite metal slab(Au)., which describes the propagation of SPs at a dielectric-metal interface $[5,29,31]$.For the second region (air- $\mathrm{Si}_{3} \mathrm{~N}_{4}-\mathrm{Au}$ ), the effective propagation constant $\left(\beta_{b}\right)$ is implicit in the next equation $[5,26,27,29-32]:$

$$
\tanh \left(\mathrm{k}_{2} \mathrm{l}_{\mathrm{y}}\right)=-\left(\frac{\varepsilon_{\mathrm{Au}} \varepsilon_{2} \mathrm{k}_{2} \mathrm{k}_{1}+\varepsilon_{1} \varepsilon_{2} \mathrm{k}_{\mathrm{Au}} \mathrm{k}_{2}}{\varepsilon_{\mathrm{Au}} \varepsilon_{1} \mathrm{k}_{2}^{2}+\varepsilon_{2}^{2} \mathrm{k}_{\mathrm{Au}} \mathrm{k}_{1}}\right)
$$

where $k_{i}=\left(\beta_{b}^{2}-\varepsilon_{i} k_{0}^{2}\right)^{1 / 2}$ is the wavenumber in each medium and the subscripts $i=1,2$ and Au represent the different materials: air, $\mathrm{Si}_{3} \mathrm{~N}_{4}$, and gold, respectively. By solving the above transcendental equation for $\beta_{b}$, the effective propagation constant of SPs traveling in the second region can be calculated. Moreover, note that $\beta_{b}$ has an explicit dependence on the height of the dielectric $\left(l_{y}\right)$. This feature has been used in the design of interesting applications such as lenses[26,27,33] and photonic nanojets[29-31]. Here we exploit this feature to design GRIN-SP dielectric steerers able to change the direction of propagation of SPs to a different output angle. Finally, the effective refractive index in each region can be calculated by the ratio of the effective propagation constant and the wavenumber in free-space $n_{S P a, b}$ $=\beta_{a, b} / k_{0}$.

The analytical results of the real and imaginary parts of the effective refractive index in region $\mathrm{b}\left(n_{S P, b}\right)$ as a function of $l_{y}$ [using equation (1)] at the design wavelength of $633 \mathrm{~nm}$ calculated following the described method are shown in figure $1(\mathrm{c})$. As observed, the real part $\left(\operatorname{Re}\left\{n_{S P, b}\right\}\right)$ can be tuned from a minimum value of 1.1 approaching asymptotically 2.43 when $l_{y}$ is modified from 0 to $200 \mathrm{~nm}$. This is an expected performance since the lower value corresponds to the effective refractive index for the air-Au region with a value of $\operatorname{Re}\left\{n_{S P, a}\right\}$ $=1.1$ while the higher value corresponds to the $\mathrm{Si}_{3} \mathrm{~N}_{4}-\mathrm{Au}$ region considering two semi-infinite media giving as a result $\operatorname{Re}\left\{n_{S P, a}\right\}=2.44$. It is important to note that the imaginary part of the effective refractive index is $\operatorname{Im}\left\{n_{S P, b}\right\}<<\operatorname{Re}\left\{n_{S P, b}\right\}$ for all the values of $l_{y}$ at the design wavelength. Moreover, since 
the thickness of the slab $l_{z}$ along the propagation axis is small (of the order of the wavelength as it will shown in the following discussions), loss does not degrade noticeably the performance of the nanostructures here designed.

Once the propagation features of SPs within the two regions have been explained, the design of the GRIN-SP steerers can be done straightforwardly using ray tracing [34-37]. Each element (position along the $x$ axis) of the GRIN-SP steerer should introduce a phase delay at its output to deflect the incident beam in an arbitrary angle. This phase delay depends on the position along the $x$ axis and the phase introduced inside of the structure and can be calculated as follows:

$\Delta \phi(x)=\bmod \left(\beta_{b}(x) l_{z}, 2 \pi\right)=\bmod \left(\beta_{0} l_{z}+\beta_{a} x \sin \theta, 2 \pi\right)(2)$

where $\bmod$ is the modulo operator, $\beta_{b}(x), \beta_{0}$ and $\beta_{a}$ are the propagation constant at each position along the $x$ axis, at the rightmost position and outside of the dielectric, respectively and $\theta$ is the output angle. By using equations (1)-(2), the phase delay and height $l_{y}$ of the $\mathrm{Si}_{3} \mathrm{~N}_{4}$ block at each position can be calculated once the output angle is fixed. By applying this design procedure, two different GRIN-SP steerers are designed to deflect the incoming SPs to an output angle of $\theta=$ $10^{\circ}$ and $\theta=30^{\circ}$. As described before, the phase introduced by the nanostructures depends on the position along the $x$ axis. Hence, in order to take into account that the fabrication of such structures may not be completely smooth, a piecewise approximation is used here and all the nanostructures are designed using discrete positions along the $x$ axis with a step of $d_{x}=\lambda_{0} / 15$ at the design wavelength of $633 \mathrm{~nm}$. With these parameters, all the GRIN-SP steerers have a total width of $(m+1) d_{x} \sim 3.55 \lambda_{0}$ along the $x$ axis with a total of $m=55$ positions. Moreover, $l_{y}$ is limited to the interval $3-60 \mathrm{~nm}$ where the phase varies significantly (larger values would lead to a negligible phase change).

Another parameter that should be considered in the designs is the length along the $z$ axis $\left(l_{z}\right)$. To fix this dimension, we allow a maximum phase advance of $2 \pi$ of the wave traveling inside the medium[38-41]. With this criterion, the length is taken as $l_{z}=\lambda_{0} / \Delta n_{S P, b}=1.14 \lambda_{0}$ where $\Delta n_{S P, b}=0.88$ is the variation of the effective refractive index between the extreme values $l_{y}=60 \mathrm{~nm}$ and $l_{y}=3 \mathrm{~nm}$.

\section{Results and Discussion}

The GRIN-SP steerers were designed following considerations described in the previous section and the results are shown in figure 2 . The magnitude of $l_{y}$ at each position along the $x$ axis for both designs is shown in figure 2(a). As observed in both cases, $l_{y}$ varies within the chosen range $(3-60 \mathrm{~nm})$. By comparing both profiles, it can be seen that for $\theta=30^{\circ}$, the profile has two variations and $l_{y}$ is changed to $\sim 56 \mathrm{~nm}$ once its previous value was close to the required minimum $\left(l_{y}=3 \mathrm{~nm}\right)$, as expected. For completeness, the values of $\operatorname{Re}\left\{n_{S P, b}\right\}$ for both designs are shown in figure 2(b) where the maximum $\left(\operatorname{Re}\left\{n_{S P, b}\right\}=1.98\right)$ and minimum limits $\left(\operatorname{Re}\left\{n_{S P, b}\right\}=1.1\right)$ are also observed.

The results of the simulated magnetic field distribution $\left(H_{x}\right)$ on the surface of the metal $(y=0)$ for both designs is shown in figure $2(\mathrm{c}, \mathrm{d})$. A clear steering of the incoming SPs is achieved with both structures. Note that, despite the fact that the local periodicity approximation has been applied in the proposed structures (where each $l_{y}$ dimension along the $x$-axis is calculated assuming an infinite $\mathrm{Si}_{3} \mathrm{~N}_{4}$ dielectric along the $x$ direction) steering SPs is always achieved. This technique has been widely used with metamaterials, demonstrating that it is a good approximation when designing GRIN structures [4246]. Moreover, note that for $\theta=30^{\circ}$, there are two other directions where the SPs are deflected. These beams correspond to the diffraction lobes that arise due to the abrupt change of $l_{y}$ at $x / \lambda_{0} \sim-1.77$ which produces a stepped profile of the nanostructure (as explained before). As observed in figure $2(d)$, they correspond to the $(-1,0)$ and $(-2,0)$ diffraction modes whose angles can be easily calculated by the wellknown equation of grating lobes[36,47,48]. An easy calculation using the periodicity of figure 2(a) gives a deflection angle of $-1.57^{\circ}$ and $-33^{\circ}$ for the first and second diffraction mode, in excellent agreement with the simulation results. Despite the fact that these lobes will always appear for long values of $\theta$, their magnitude is lower than the main lobe at the design angle [see figure 2(d)]. This demonstrates that, by simply and properly tailoring the height of a dielectric on top of a metal, it is possible to deflect the SPs.

The designs shown in figure 2 were done by fixing the thickness $\left(l_{z}\right)$ of the $\mathrm{Si}_{3} \mathrm{~N}_{4}$ dielectric block and changing its height $\left(l_{y}\right)$ in order to achieve the required effective refractive index and phase delay. However, the opposite scenario is also possible, i.e., we can fix $l_{y}$ and change $l_{z}$ to deflect SPs. Note that with this option, the structure is no longer GRIN because $\operatorname{Re}\left\{n_{S P, b}\right\}$ is the same for all locations along the $z$ axis. To verify this, two SP steerers were designed with the same output angles as before $\left(\theta=10^{\circ}\right.$ and $\left.\theta=30^{\circ}\right)$. Here, a height of $l_{y}=1 \mu \mathrm{m}$ is chosen to work with the maximum effective refractive index of 2.43 and hence minimize the thickness of the structure (the phase advance is proportional to the effective refractive index).

With these considerations, the values of $l_{z}$ at each position along the $x$ axis for each design are shown in figure 3(a). As observed, similar profiles to those shown in figure 2(a) are obtained with two steps for the design with $\theta=30^{\circ}$. The results of the magnetic field distribution $\left(H_{x}\right)$ on the surface of the metal $(y=0)$ for both designs are shown in figure $3(b, c)$. As observed, both structures are able to deflect the incoming SPs at the required output angle. Again, for the design with $\theta=$ $30^{\circ}$, a sidelobe is observed at $-0.98^{\circ}$ which corresponds to the $(-1,0)$ diffraction order. 

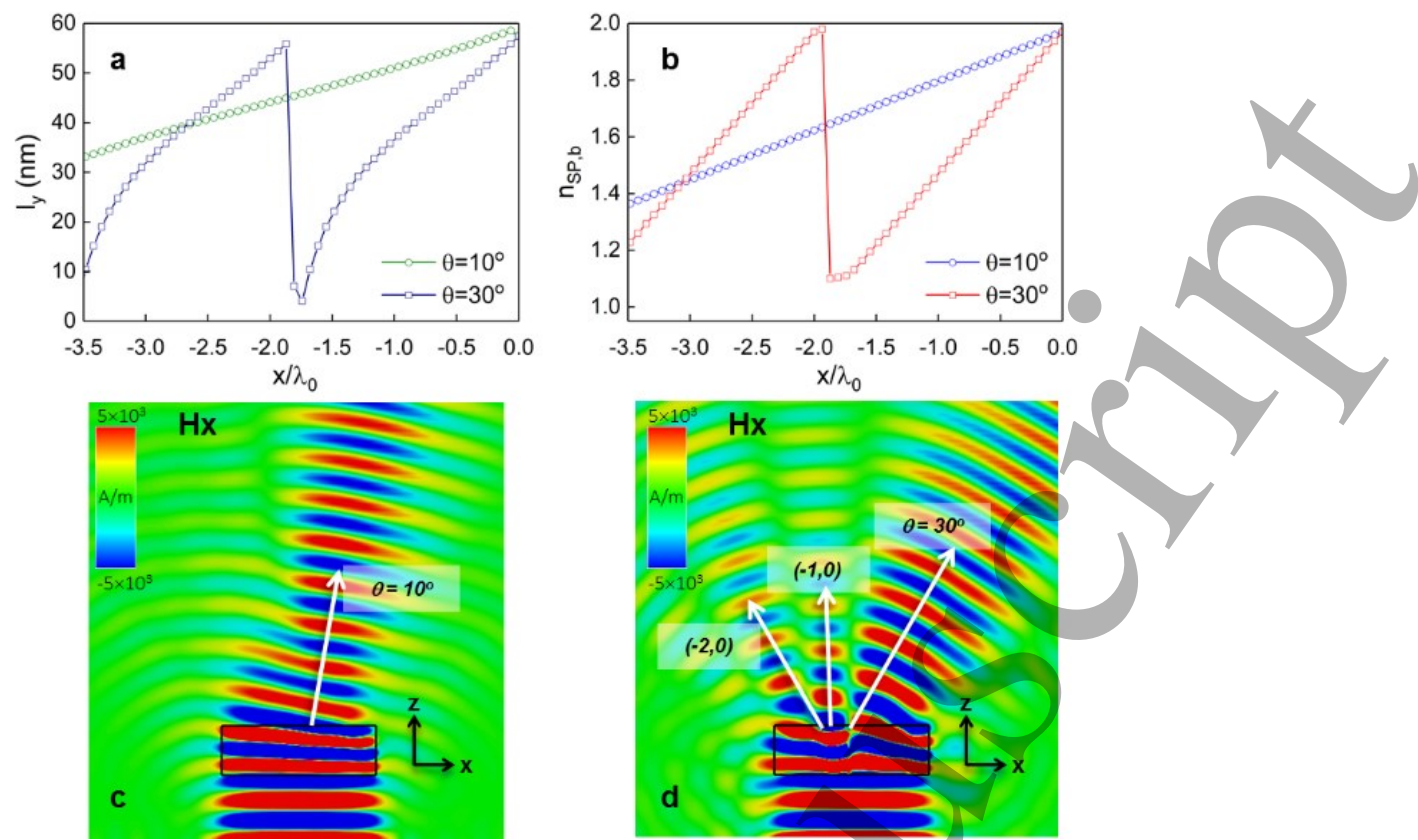

Figure. 2. (a) Analytical results values of $l_{y}$ at each position along the $x$ axis for the GRIN-SP steerers designed to deflect the incoming SPs to an output angle of $\theta=10^{\circ}$ (green) and $\theta=30^{\circ}$ (blue). (b) Effective refractive index along the $x$ axis for the designs with $\theta=10^{\circ}$ (blue) and $\theta=30^{\circ}$ (red). Magnetic field distribution $H_{x}$ at $y=0$ (surface of the metal) for the designs with (c) $\theta=10^{\circ}$ (blue) and (d) $\theta=30^{\circ}$.

In order to fully assess the performance of the designed steerers a deep study considering output angles ranging from $0^{\circ}$ to $60^{\circ}$ was carried out. Both strategies, i.e. changing $l_{y}$ and changing $l_{z}$, were evaluated. The magnetic field $H_{x}$ was calculated along a semicircle of radius $r=6.5 \lambda_{0}$ from the output surface of the GRIN-SP steerers (changing $l_{y}$ ) and SP steerers (changing $l_{z}$ ) in order to record the angular distribution of the deflected SPs (see figure 4(b) which shows the field distribution along the semicircle for a designed output angle of $10^{\circ}$ ). With this configuration, the magnitude of $H_{x}$ at each output angle normalized to the case of an output angle of $0^{\circ}$ is shown in figure 4(a). As observed, the magnitude decreases as the output angle increases. As shown in figure 2 and figure 3, the number of steps in the profile of the nanostructures increases when increasing $\theta$. Hence, the SPs will be also deflected to other angles related to higher order diffraction modes, reducing the $H_{x}$ of the SPs at the designed output angle. Moreover, by comparing the results with both designs, it is observed that the performance is better when $l_{y}$ is varied. In this case, the magnetic field magnitude decays to half its maximum at $\theta=60^{\circ}$, whereas with the second strategy this happens for $\theta=30^{\circ}$. This can be explained by observing that for large $\theta$ the number of steps in the profile increases in both cases. Hence, in the second strategy the propagating SPs penetrate into the stepped dielectric (for angles $>30^{\circ}$ ) zones along the $z$ axis producing a shadowing effect of the SPs which
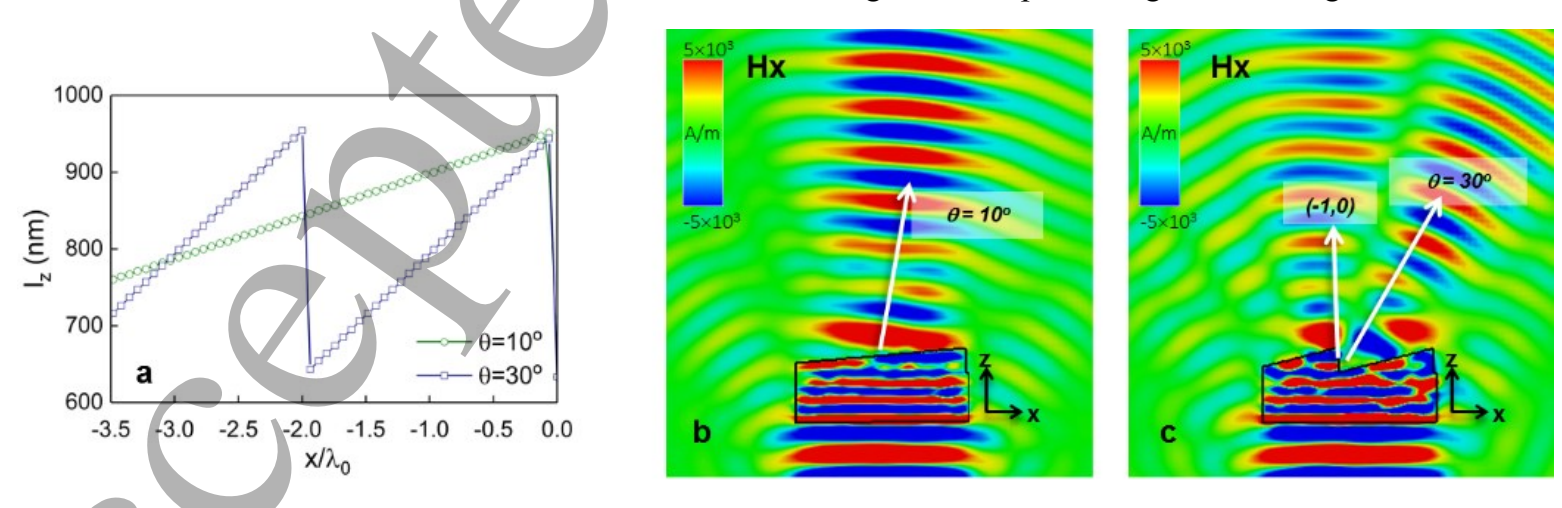

Figure 3. (a) Analytical values of $l_{z}$ along the $x$ axis for the SP steerers designed for $\theta=10^{\circ}$ (green) and $\theta=30^{\circ}$ (blue). Magnetic field distribution $H_{x}$ at $y$ $=0$ for the designs with (b) $\theta=10^{\circ}$ and (c) $\theta=30^{\circ}$. 

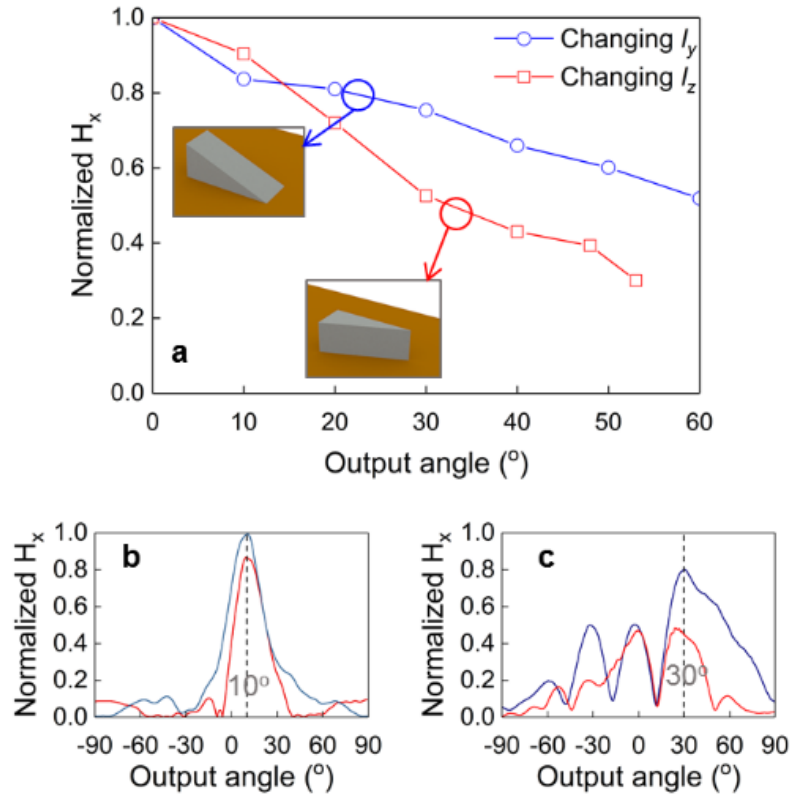

Figure 4. (a) Normalized magnitude of $H_{x}$ for several GRIN-SP steerer designed with $\theta$ ranging from $0^{\circ}$ to $60^{\circ}$ changing $l_{y}$ (blue) and SPsteerers changing $l_{z}$ (red). An artistic representation for both cases is shown as insets to guide the eye. Normalized magnitude of $H_{x}$ for the designs changing $l_{y}$ (blue) and changing $l_{z}$ (red) considering an output angle of (b) $10^{\circ}$ and (c) $30^{\circ}$ calculated along a semicircle of radius $r=$ $6.5 \lambda_{0}$ from the output surface of the GRIN-SP and SP - steerers.

is translated into a reduced amplitude in the direction of the designed output angle. Also, note that with these designs the output angle is slightly deviated from the designed ones for angles larger than $50^{\circ}$. This is in sharp contrast with the case when $l_{y}$ is changed because the output profile is flat and there is no patterning of the dielectric along the $z$ axis, making this strategy more robust in terms of the decay of the amplitude of the SPs for longer designed angles. It is important to note that the two variations (or steps) of $l_{y}$ and $l_{z}$ shown in figure $2 \mathrm{a}$ and figure $3 \mathrm{a}$ for the designs with $\theta=30^{\circ}$ may be reduced by using a thick dielectric along the $z$ axis. However, this would result in different total dimensions for all the designs and the comparison of their performance would not be completely fair.

For the sake of completeness, the spectral response of all the GRIN-SP and SP steerers was also obtained to assess their operation bandwidth. Here, we consider the nanostructures shown in figure 2 and figure 3 for designed output angles of $\theta$ $=10^{\circ}$ and $\theta=30^{\circ}$. The results of the output angle as a function of the incident SPs at different wavelengths for the designs changing $l_{y}$ and $l_{z}$ are shown in Figure 5(a,b), respectively. From these results, again changing $l_{y}$ leads to a more robust performance in terms of the output angle with a smooth change of the output angle around the design wavelength. A relatively broad operation bandwidth (considering the spectral range where the output angle is deviated $10 \%$ from the
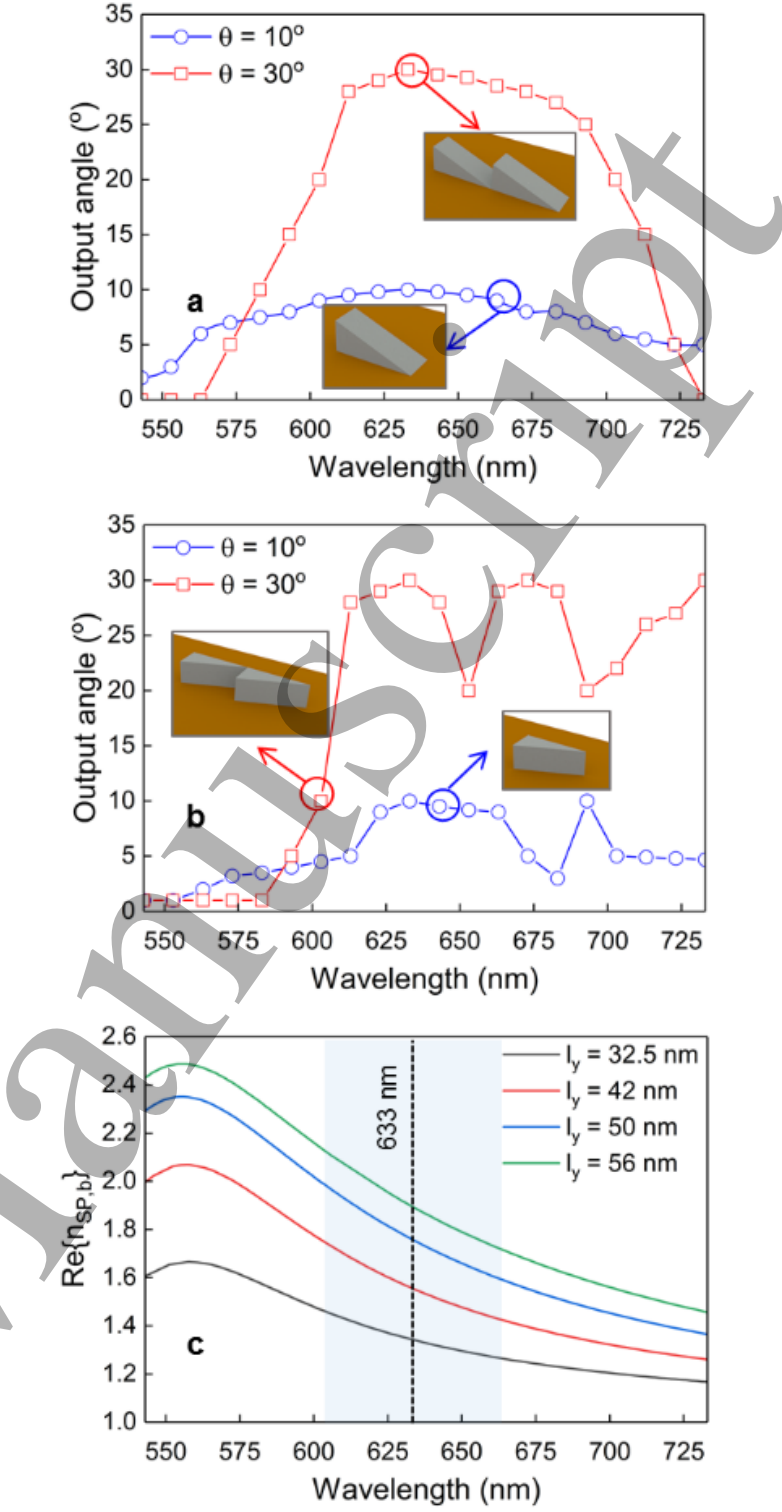

Figure 5. Output angle as a function of the incident wavelength for the nanostructures: (a) changing $l_{y}$ and (b) changing $l_{z}$ considering $\theta=10^{\circ}$ (blue) and $\theta=30^{\circ}$ (red). The schematic representations of each design are shown as insets in each panel for clarity. (c) Analytical results of the $n_{S P, b}$ for the design with changing $l_{y}$ considering four intermediate locations along the $x$-axis for $\theta=10^{\circ}$ with $l_{y}=32.5 \mathrm{~nm}$ (black), $l_{y}=42$ $\mathrm{nm}$ (red), $l_{y}=50 \mathrm{~nm}$ (blue) and $l_{y}=56 \mathrm{~nm}$ (green). The design frequency is shown as black dotted line along with the bandwidth represented as the blue region.

designed value) is obtained with a value of $\sim 60 \mathrm{~nm}$ and $\sim 55$ $\mathrm{nm}$ for $\theta=10^{\circ}$ and $\theta=30^{\circ}$, respectively. Changing $l_{z}$ has a poorer performance and in these designs, a bandwidth of $\sim 35$ $\mathrm{nm}$ and $\sim 20 \mathrm{~nm}$ for $\theta=10^{\circ}$ and $\theta=30^{\circ}$ is obtained. Note that, despite the fact that both configurations have different robustness in terms of the incident wavelength, their bandwidth is still relatively high considering that the structures were designed for an optimal operation at a single 
wavelength. This is because of the smooth variation of the $n_{S P, b}$ around the designed value (see figure $5 \mathrm{c}$ ). For instance, for the design with $\theta=10^{\circ}, n_{S P, b}$ is varied $\sim 7 \%$ and $\sim 7.9 \%$ at the two extreme locations along the $x$-axis with $l_{y}=32.5 \mathrm{~nm}$ and $l_{y}=56 \mathrm{~nm}$, respectively (see figure $2 \mathrm{a}$ for the positions along $x$ and figure $5 \mathrm{c}$ for the $n_{S P, b}$ values within the spectral range under study). Similar performance is observed for the case with $\theta=30^{\circ}$ since the values of $l_{y}$ shown in figure $5 \mathrm{c}$ are also valid for this case. For the designs with fixed $l_{y}$ and changing $l_{z}$, the bandwidth is reduced because of the shadowing effect experienced by the SPs along the propagation axis, as described before. Finally, it is important to highlight that the GRIN-SP steerers here proposed can be designed at any optical wavelength. For instance, several designs at $1000 \mathrm{~nm}$ were also studied (not shown here) with the ability to deflect the incoming SPs. It is important to note that reflections are present in the structures because of the values of $\operatorname{Re}\left\{n_{S P, b}\right\}$ and $\operatorname{Re}\left\{n_{A u}\right\}$. However, they are slightly reduced for the GRIN-SP steerers compared to the SP steerers because of the smooth change of $\operatorname{Re}\left\{n_{S P, b}\right\}$. For instance, it is it is $|\Gamma|=0.43$ and $|\Gamma|=0.49$ for the designs changing $l_{y}$ and $l_{z}$, respectively considering an output angle of $30^{\circ}$.

\section{Conclusions}

To sum up, the design and study of several GRIN-SP structures with the ability to steer the direction of propagation of SPs has been presented. The design procedure has been shown in terms of the effective refractive index achieved when a dielectric with a certain height is placed in between two semi-infinite media (air and metal). Several designs (for output angles ranging from $\theta=10^{\circ}$ to $\theta=60^{\circ}$ ) have been proposed by tailoring the height of the dielectric and fixing its dimension along the propagation direction and compared with SP-steerers designed with a fixed height while changing the length of the dielectric. The nanostructure designs have been evaluated numerically demonstrating a good agreement with the analytical calculations showing their capability to deflect the propagation of the incoming SPs at the required output angle. The robustness of the structures has been evaluated in terms of their spectral response demonstrating that they can achieve beam steering of the SPs for wavelengths $\sim 60 \mathrm{~nm}$ around the designed value. The results here presented may be used in optical applications where the guiding and full control of the direction of SPs is required opening a new path on the control and manipulation of SP propagation.

\section{Acknowledgements}

V.P.-P. is supported by the Newcastle University (Newcastle University Research Fellow). M. B. acknowledges support by the Spanish Ministerio de Economía y Competitividad with European Union FEDER funds [TEC2014-51902-C2-2-R].

\section{References}

[1] Shen Y, Friend C S, Jiang Y, Jakubczyk D, Swiatkiewicz J and Prasad P N 2000 Nanophotonics: Interactions, Materials, and Applications J. Phys. Chem. B 104 7577-87

[2] Chien Y H, Wang C H, Liu C C, Chang S H, Kong K V and Chang Y C 2017 Large-Scale Nanofabrication of Designed Nanostructures Using Angled Nanospherical-Lens Lithography for Surface Enhanced Infrared Absorption Spectroscopy ACS Appl. Mater. Interfaces 9 24917-25

[3] Wu H, Li C, Zhao Z, Li H and Jin Y 2016 Free-Standing Monolayered Metallic Nanoparticle Networks as Building Blocks for Plasmonic Nanoelectronic Junctions ACS Appl. Mater. Interfaces 8 1594-9

[4] Krasavin A V. and Zayats A V. 2015 Active Nanophotonic Circuitry Based on Dielectric-loaded Plasmonic Waveguides Adv. Opt. Mater. 3 1662-90

[5] S. A. Maier 2007 Plasmonics: Fundamentals and Applications (Springer)

[6] Knight M W, King N S, Liu L, Everitt H O, Nordlander P and Halas N J 2014 Aluminum for plasmonics ACS Nano 8 834-40

[7] Pitarke J M, Silkin V M, Chulkov E V and Echenique P M 2006 Theory of surface plasmons and surface-plasmon polaritons Rep. Prog. Phys. 154

[8] Liu Z, Steele J M, Srituravanich W, Pikus Y, Sun C and Zhang X 2005 Focusing surface plasmons with a plasmonic lens. Nano Lett. 5 1726-9

[9] Yin L, Vlasko-Vlasov V K, Pearson J, Hiller J M, Hua J, Welp U, Brown D E and Kimball C W 2005 Subwavelength focusing and guiding of surface plasmons Nano Lett. 5 1399-402

[10] Feng L, Tetz K, Slutsky B, Lomakin V and Fainman Y 2008 Fourier plasmonics: Diffractive focusing of in-plane surface plasmon polariton waves Appl. Phys. Lett. 91 081101-1-

Tanemura T, Balram K C, Ly-Gagnon D S, Wahl P, White J S, Brongersma M L and Miller D A B 2011 Multiplewavelength focusing of surface plasmons with a nonperiodic nanoslit coupler Nano Lett. 11 2693-8

[12] Long Y, Zhang Z and Su X 2016 Robust subwavelength focusing of surface plasmons on graphene epl $\mathbf{1 1 6}$

[13] Zhang Z, Zhang L, Li H and Chen H 2014 Plasmon induced transparency in a surface plasmon polariton waveguide with a comb line slot and rectangle cavity Appl. Phys. Lett. 104

[14] Bozhevolnyi S I, Erland J, Leosson K, Skovgaard P M W and Hvan J M 2001 Waveguiding in surface plasmon polariton band gap structures Phys. Rev. Lett. $863008-11$

[15] Zhang Z, Long Y, Ma P and Li H 2017 Tunable highchannel-count bandstop graphene plasmonic filters based on plasmon induced transparency Nanotechnology 28 1-6

[16] Roxworthy B J, Ko K D, Kumar A, Fung K H, Chow E K C, Liu G L, Fang N X and Toussaint K C 2012 Application of plasmonic bowtie nanoantenna arrays for optical trapping, stacking, and sorting Nano Lett. 12 796-801

[17] Navarro-Cia M and Maier S A 2012 Broad-band nearinfrared plasmonic nanoantennas for higher harmonic generation. ACS Nano 6 3537-44

[18] Pacheco-Peña V, Beruete M, Fernández-Domínguez A I, Luo Y and Navarro-Cía M 2016 Description of bow-tie nanoantennas excited by localized emitters using conformal transformation ACS Photonics 3 1223-32

[19] Pacheco-Peña V, Fernández-Domínguez A I, Luo Y, Beruete M and Navarro-Cía M 2017 Aluminum Nanotripods for Light-Matter Coupling Robust to Nanoemitter Orientation Laser Photon. Rev. 17000511700051 
[20]

Pacheco-Peña V and Navarro-Cía M 2018 Understanding quantum emitters in plasmonic nanocavities with conformal transformation: Purcell enhancement and forces Nanoscale 10 $13607-16$

[21] Genevet P, Wintz D, Ambrosio A, She A, Blanchard R and Capasso F 2015 Controlled steering of Cherenkov surface plasmon wakes with a one-dimensional metamaterial Nat. Nanotechnol. 10 804-9

[22] Liu W 2017 Controllable Steering and Tuning of Surface Plasmons on the Metallic Nano-film with Nanoslits Array Plasmonics $1-5$

[23] Wang X, Deng Y, Li Q, Huang Y, Gong Z, Tom K B and Yao J 2016 Excitation and propagation of surface plasmon polaritons on a non-structured surface with a permittivity gradient Light Sci. Appl. 5 1-6

[24] Grajower M, Lerman G M, Goykhman I, Desiatov B, Yanai A, Smith D R and Levy U 2013 Subwavelength plasmonics for graded-index optics on a chip. Opt. Lett. 383492 5

[25] Liu Y, Zentgraf T, Bartal G and Zhang X 2010 Transformational plasmon optics Nano Lett. 10 1991-7

[26] Smolyaninova V N, Smolyaninov I I, Kildishev A V and Shalaev V M 2010 Maxwell fish-eye and Eaton lenses emulated by microdroplets. Opt. Lett. 35 3396-8

[27] Zentgraf T, Liu Y, Mikkelsen M H, Valentine J and Zhang X 2011 Plasmonic Luneburg and Eaton lenses. Nat. Nanotechnol. 6 151-5

[28] Johnson P B and Christry R W 1972 Optical Constants of the Noble Metals Phys. Rev. B 6 4370-9

[29] Pacheco-Peña V, Minin I V, Minin O V and Beruete M 2016 Comprehensive analysis of photonic nanojets in 3D dielectric cuboids excited by surface plasmons Ann. Phys. June

[30] Pacheco-peña V, Minin I V, Minin O V and Beruete M 2016 Increasing Surface Plasmons Propagation via Photonic Nanojets with periodically spaced 3D dielectric cuboids Photonics 3 1-7

[31] Ju D, Pei H, Jiang Y and Sun X 2013 Controllable and enhanced nanojet effects excited by surface plasmon polariton Appl. Phys. Lett. 102171109

[32] Pacheco-Pena V 2016 Metamaterials and plasmonics applied to devices based on periodic structures at high frequencies: microwaves, terahertz and optical range (Public University of Navarra)

[33] Bezus E A, Doskolovich L L, Kazanskiy N L, Soifer V a and Kharitonov S I 2010 Design of diffractive lenses for focusing surface plasmons $J$. Opt. 12 1-7

[34] Pacheco-Peña V, Torres V, Beruete M, Navarro-Cía M and Engheta N $2014 \epsilon$-near-zero (ENZ) graded index quasioptical devices: steering and splitting millimeter waves J. Opt. 16 094009

[35] Pacheco-Peña V, Navarro-Cía M and Beruete M 2016 Epsilon-near-zero metalenses operating in the visible Opt. Laser Technol. 80 162-8

[36] Born M and Wolf E 1999 Principles Of Optics (New York: Cambridge University Press)

[37] Pacheco-Pena V, Torres V, Navarro-Cia M, Beruete M, Sorolla M and Engheta N 2013 \&-near-zero Graded Index structure as a bi-concave metallic lens using stacked rectangular near cut-off waveguides 2013 7th European Conference on Antennas and Propagation, EuCAP 2013

[38] Pacheco-Peña V, Orazbayev B, Torres V, Beruete M and Navarro-Cía M 2013 Ultra-compact planoconcave zoned metallic lens based on the fishnet metamaterial Appl. Phys. Lett. 103183507
[39] Pacheco-Peña V, Navarro-Cía M, Orazbayev B, Minin I V., Minin O V. and Beruete M 2015 Zoned Fishnet Lens Antenna With Reference Phase for Side-Lobe Reduction IEEE Trans. Antennas Propag. 63 3710-4

[40] Hristov H D 2000 Fresnel Zones in Wireless Links, Zone Plate Lenses and Antennas (Inc., Norwood, MA: Artech House)

[41] Pacheco-Pena V, Minin I V., Minin O V. and Beruete M 2018 Phase reversal technique applied to fishnet metalenses Int. J. Antennas Propag.

[42] Li Y B, Cai B G, Cheng Q and Cui T J 2015 Surface Fourier-transform lens using a metasurface J. Phys. D. Appl. Phys. 48

[43] Neu J, Krolla B, Paul O, Reinhard B, Beigang R and Rahm M 2010 Metamaterial-based gradient index lens with strong focusing in the THz frequency range Opt. Express 18 27748-57

[44] Smith D, Mock J, Starr a. and Schurig D 2005 Gradient index metamaterials Phys. Rev. E 71036609

[45] Torres V, Pacheco-Peña V, Rodríguez-Ulibarri $P$, Navarro-Cía M, Beruete M, Sorolla M and Engheta N 2013 Terahertz epsilon-near-zero graded-index lens Opt. Express 21 9156-66

[46] Pacheco-Peña V, Engheta N, Kuznetsov S, Gentselev A and Beruete M/2017 Experimental Realization of an EpsilonNear-Zero Graded-Index Metalens at Terahertz Frequencies Phys. Rev. Appl. 8034036

[47] Pacheco-Peña V, Orazbayev B, Beaskoetxea U, Beruete M and Navarro-Cía M 2014 Zoned near-zero refractive index fishnet lens antenna: Steering millimeter waves J. Appl. Phys. 115 124902-1-8

[48] Rodriguez-Ulibarri P, Pacheco-Peña V, Navarro-Cia M, Serebryannikov A E and Beruete M 2015 Experimental demonstration of deflection angle tuning in unidirectional fishnet metamaterials at millimeter-waves Appl. Phys. Lett. 106061109 $1-5$ 